CLINICAL STUDY

\title{
Decrease of brachial-ankle pulse wave velocity in female subclinical hypothyroid patients during normalization of thyroid function: a double-blind, placebo-controlled study
}

Toshiki Nagasaki, Masaaki Inaba, Shinsuke Yamada, Kumi Shirakawa, Yuki Nagata, Yasuro Kumeda, Yoshikazu Hiura, Hideki Tahara, Eiji Ishimura and Yoshiki Nishizawa

Department of Metabolism, Endocrinology and Molecular Medicine, Internal Medicine, Osaka City University Graduate School of Medicine, 1-4-3, Asahi-machi, Abeno-ku, Osaka-City 545-8585, Japan

(Correspondence should be addressed to M Inaba; Email: inaba-m@med.osaka-cu.ac.jp)

\begin{abstract}
Objective: Subclinical hypothyroidism affects $5-15 \%$ of the general population, is especially prevalent in females, and may be associated with increased morbidity from cardiovascular disease, although it remains controversial. We recently reported a significant increase in the brachial-ankle pulse wave velocity (baPWV), a parameter of arterial stiffening and an independent predictor of cardiovascular events, in subclinical hypothyroidism without thyroiditis. The current study was performed to assess changes in baPWV in female subclinical hypothyroidism with autoimmune chronic thyroiditis (Hashimoto's disease) after restoration of normal thyroid function.

Methods: In a randomized placebo-controlled study, 95 female subclinical hypothyroid patients were monitored for changes in baPWV before and after levothyroxine $\left(\mathrm{L}-\mathrm{T}_{4}\right)$ replacement therapy. Changes in baPWV were also measured in 42 age-matched normal female subjects.

Results: The baseline baPWV values in patients with subclinical hypothyroidism were significantly higher than in normal subjects. With attainment of euthyroidism, baPWV showed a significant decrease from $1776.7 \pm 86.0$ to $1674.3 \pm 79.2 \mathrm{~cm} / \mathrm{s}(P=0.006)$ in patients treated with $\mathrm{L}_{-} \mathrm{T}_{4}$, but the changes in baPWV and TSH were not correlated. The change in baPWV was significantly and negatively correlated with age and baseline pulse pressure, but multiple regression analysis revealed that these parameters failed to be associated with the change in baPWV.

Conclusions: Sustained normalization of thyroid function during $\mathrm{L}_{-} \mathrm{T}_{4}$ replacement therapy significantly decreases baPWV in female subclinical hypothyroid patients with autoimmune chronic thyroiditis, suggesting the improvement of arterial stiffening and, consequently, possible prevention of cardiovascular disease.
\end{abstract}

European Journal of Endocrinology $160409-415$

\section{Introduction}

Subclinical hypothyroidism occurs in $5-15 \%$ of the population, is highly prevalent in women over 60 years of age (1), and may be a risk factor for aortic atherosclerosis and myocardial infarction $(2,3)$, although it remains controversial. Accelerated atherosclerosis in subclinical hypothyroidism occurs due to multiple mechanisms, including increased diastolic blood pressure (DBP) (4) and dyslipidemia (5, 6). Recent studies have shown that patients with subclinical hypothyroidism have a higher intima-media thickness (IMT) of the carotid artery, and increased left ventricular dysfunction at rest and systolic dysfunction on effort, which are risk factors for both atherosclerosis and myocardial infarction $(7,8)$. These factors can be improved by normalization of thyroid function with levothyroxine $\left(\mathrm{L}-\mathrm{T}_{4}\right)$ replacement therapy $(7,8)$.

We recently found a significant increase in brachialankle pulse wave velocity (baPWV), a parameter of arterial stiffness (9) and an independent predictor of cardiovascular mortality (10), in male and female subclinical hypothyroid patients without thyroiditis (11). Moreover, baPWV is more likely to decrease in females than males after normalization of thyroid function by $\mathrm{L}-\mathrm{T}_{4}$ replacement therapy (12), although not in a placebo-controlled study. This background prompted us to examine $\mathrm{L}_{-} \mathrm{T}_{4}$ replacement therapy in female patients with subclinical hypothyroidism due to autoimmune chronic thyroiditis to determine i) whether baPWV might decrease after normalization of thyroid function and ii) which of a number of clinical variables 
including blood pressure, lipid profiles, thyroid hormone level, and pre-ejection time (PET)/left ventricular ejection time (ET) ratio, a parameter of systolic dysfunction reported to be increased in subclinical hypothyroidism (8), has the greatest influence on baPWV during $\mathrm{L}_{-} \mathrm{T}_{4}$ replacement.

\section{Patients and methods}

The study was approved by the ethical committee of Osaka City University Hospital. Written informed consent was obtained from each patient. Ninety-five consecutive patients with newly detected subclinical hypothyroidism due to chronic thyroiditis with antithyroglobulin or antithyroid peroxidase antibodies were enrolled during the 24-month period from June 2005 to May 2007 and randomly assigned to receive either $\mathrm{L}_{-} \mathrm{T}_{4}$ replacement therapy $(n=48)$ or identical placebo tablets $(n=47)$ in a blinded manner. The duration of the therapy was $4.86 \pm 0.20$ months, which is short but sufficiently long to assess the change in baPWV (13).

Diagnosis of subclinical hypothyroidism was established based on the elevation of serum TSH to above the normal upper limit, with normal levels of serum free thyroxine $\left(\mathrm{FT}_{4}\right)$ and free triiodothyronine $\left(\mathrm{FT}_{3}\right)$. To confirm sustained subclinical hypothyroidism, thus excluding patients with a temporary condition such as that in recovery from a non-thyroidal illness, measurement of TSH was conducted at least twice (mean: 2.03 times) for 6 months before treatment. Moreover, to avoid confounding factors known to affect atherosclerosis, patients suffering from major diseases such as hypertension (13), hyperlipidemia, diabetes mellitus or patients receiving other hormone replacement therapy (14) or taking any drugs that affect the lipid profile and atherosclerosis such as antihypertensive agents (13, 15), lipid-lowering drugs (16), anti-platelet drugs (17), and bisphosphonates including etidronate (18) were excluded from the study.

Normal control subjects who joined the Health-Check Program at Osaka City University Hospital were enrolled consecutively as age-matched controls and monitored for an average of $4.80 \pm 0.24$ months. Normal controls had no history of thyroid disease, had neither goiter nor an autoimmune-antibody titer, and were in a euthyroid state.

The smoking index (daily number of cigarettes multiplied by the number of years of smoking) between subclinical hypothyroid patients and normal controls did not differ significantly and during this study individual smoking habit did not change.

\section{Protocol for $\mathrm{L}_{-} \mathrm{T}_{4}$ replacement therapy}

Female subclinical hypothyroid patients in an untreated state were administered $\mathrm{L}_{-} \mathrm{T}_{4}$ at an initial daily dose of $12.5 \mu \mathrm{g}$ and serum levels of $\mathrm{FT}_{4}, \mathrm{FT}_{3}$, and $\mathrm{TSH}$ were checked every 4 weeks. When serum TSH was still above the normal upper limit, the dose of $\mathrm{L}_{-} \mathrm{T}_{4}$ was increased serially to $18.75 \mu \mathrm{g} /$ day, $25 \mu \mathrm{g} /$ day, and maximally to $37.5 \mu \mathrm{g} /$ day until TSH normalized (the average dose was $25.8 \mu \mathrm{g} /$ day). The average length of treatment to achieve normal TSH level was $13.4 \pm 0.86$ weeks since the onset of the $\mathrm{L}_{-} \mathrm{T}_{4}$ replacement therapy, and the duration the patients had kept euthyroidism was $7.46 \pm 0.69$ weeks before the second measurement of baPWV. None of the patients experienced side effects such as arrhythmia, angina pectoris, or hypertension that would have required withdrawal or reduction of the dose of $\mathrm{L}_{-} \mathrm{T}_{4}$. Patients taking placebo completed an identical protocol, with some given additional placebo tablets to maintain the blindness of the study.

\section{Serum parameters}

Blood was drawn just before performing ultrasonography after an overnight fast. Total cholesterol, triglyceride, and high-density lipoprotein (HDL) cholesterol levels were determined using an autoanalyzer, and the low-density lipoprotein (LDL) cholesterol level was calculated according to the formula of Friedewald et al. (19). C-reactive protein (CRP) was measured using commercially available automated MAB, solid-phase, sandwich-type, enzyme immunoassay kits (Abbott Laboratories) (20). Commercially available high-capacity, random access immunoassay kits were used to measure $\mathrm{FT}_{4}, \mathrm{FT}_{3}$, and $\mathrm{TSH}$ levels (Chiron Diagnostics Co., Walpole, MA, USA) (21). Antithyroglobulin (Cosmic Co., Tokyo, Japan) and antithyroid peroxidase antibodies (Cosmic Co.) were also determined using commercially available kits (22). Highly sensitive RIA system for antithyroid antibodies was employed in the present study. In this system, highly purified thyroglobulin and thyroid peroxidase were used as antigens. The serum concentrations of anti-thyroglobulin and antithyroid peroxidase antibodies more than 0.4 and $0.3 \mathrm{U} / \mathrm{ml}$ respectively were considered positive.

\section{Pulse wave velocity}

An automatic waveform analyzer (model BP-203RPE; Colin Co., Komaki, Japan) was used to measure pulse wave velocity (PWV) simultaneously with blood pressure, electrocardiogram, and heart sounds, as described previously $(9,11)$. The reproducibility of the baPWV measurements was evaluated by repeating the measurements in 17 healthy subjects on two different occasions. The BP-203RPE analyzer measures baPWV on both sides simultaneously. Since the average coefficient of variation for baPWV was lower on the right side $(1.7 \%)$ than on the left side $(2.2 \%)$, baPWV on the right side was used as the representative value. The $\mathrm{PET} / \mathrm{ET}$ ratio was calculated using the same automatic waveform analyzer $(11,23)$. 


\section{Statistical analysis}

Data are shown as means \pm s.e.m. unless otherwise indicated. Statistical analysis was performed with statistics software (StatView version 5.0, SAS Institute, Cary, NC, USA). Differences in clinical factors among $\mathrm{L}-\mathrm{T}_{4}$-treated patients and placebo patients and normal controls were examined using the two-tailed multiple $t$-test with Bonferroni correction. The difference in the pre-/postmenopausal ratio was analyzed by $\chi^{2}$-test. The difference in mean values before and after normalization of thyroid function was assessed by a two-tailed Student's $t$-test for paired data. Spearman's rank correlation analysis was used to examine the relationships between the change in baPWV and other factors. Stepwise multiple regression analysis with forward elimination was performed to assess independent variables associated with the change in baPWV, with the $F$ value set at 4.0 at each step. $P<0.05$ were considered to be statistically significant.

\section{Results}

\section{Baseline values of baPWV and clinical variables in patients treated with $L_{-} T_{4}$ or placebo and in normal controls}

The baseline values of baPWV and other factors in subclinical hypothyroid patients and normal controls are shown in Table 1. All patients had serum TSH levels above the normal upper limit (7.25 \pm 0.69 in $\mathrm{L}_{-} \mathrm{T}_{4}$-treated patients versus $2.52 \pm 0.30$ in normal controls, $P<0.001$ ), with serum $\mathrm{FT}_{4}$ and $\mathrm{FT}_{3}$ within the respective normal ranges. There were no significant differences in age, pre-/postmenopausal ratio (6/42 in $\mathrm{L}_{-} \mathrm{T}_{4}$-treated patients, $5 / 42$ in placebos, 5/43 in normal controls), body mass index (BMI), and smoking index, systolic blood pressure (SBP) and DBP, pulse pressure and pulse rate, and PET/ET ratio among the three groups. Serum levels of total cholesterol, LDL and HDL cholesterol, triglyceride, and CRP did not differ significantly among the three groups. BaPWV was significantly higher in subclinical hypothyroid patients than in normal controls (1776.7 \pm 86.0 in $\mathrm{L}^{-} \mathrm{T}_{4}$-treated patients versus $1414.2 \pm 58.6 \mathrm{in}$ normal controls, $P<0.001)$, as we have reported previously (11).

The means \pm s.D. of smoking index were $148 \pm 402$, $108 \pm 742$, and $98 \pm 368$ in $\mathrm{L}^{-} \mathrm{T}_{4}$-treated patients, placebo patients, and normal controls respectively.

\section{Correlation between baseline baPWV and other parameters in subclinical hypothyroid patients and normal subjects}

The baPWV was significantly positively correlated with age, SBP and DBP, and pulse pressure in both subclinical hypothyroid patients and normal control subjects as we reported previously (11) (data not shown). In both groups, baPWV was not significantly correlated with $\mathrm{FT}_{3}, \mathrm{FT}_{4}$, and TSH, total, HDL, and LDL cholesterol, LDL/HDL ratio, CRP, and the PET/ejection time ratio.

Table 1 Baseline characteristics of subclinical hypothyroid patients and normal controls.

\begin{tabular}{|c|c|c|c|c|}
\hline & L-T ${ }_{4}$-treated patients & Placebo patients & Normal controls & $\boldsymbol{P}$ \\
\hline Number of subjects & 48 & 47 & 48 & \\
\hline Age (years) & $64.4 \pm 2.59$ & $66.0 \pm 3.0$ & $63.7 \pm 2.85$ & ns \\
\hline Body mass index $\left(\mathrm{kg} / \mathrm{m}^{2}\right)$ & $22.0 \pm 0.48$ & $22.2 \pm 0.51$ & $21.7 \pm 0.38$ & ns \\
\hline Smoking index & $148 \pm 58.1$ & $108 \pm 81.3$ & $98 \pm 38.9$ & ns \\
\hline Systolic BP (mmHg) & $132.8 \pm 3.9$ & $133.1 \pm 3.4$ & $131.2 \pm 3.6$ & ns \\
\hline Diastolic BP (mmHg) & $74.3 \pm 2.9$ & $75.7 \pm 1.9$ & $73.3 \pm 1.7$ & $\mathrm{~ns}$ \\
\hline Pulse pressure $(\mathrm{mmHg})$ & $58.5 \pm 2.5$ & $57.4 \pm 2.2$ & $58.3 \pm 2.3$ & ns \\
\hline Pulse rate $(/ \mathrm{min})$ & $73.4 \pm 1.9$ & $72.1 \pm 2.2$ & $70.1 \pm 2.2$ & $\mathrm{~ns}$ \\
\hline Total chol (mmol/l) & $5.59 \pm 0.27$ & $5.53 \pm 0.25$ & $5.58 \pm 0.22$ & $\mathrm{~ns}$ \\
\hline Triglyceride $(\mathrm{mmol} / \mathrm{l})$ & $1.34 \pm 0.14$ & $1.37 \pm 0.13$ & $1.75 \pm 0.4$ & $\mathrm{~ns}$ \\
\hline LDL-C (mmol/l) & $3.58 \pm 0.22$ & $3.56 \pm 0.20$ & $3.41 \pm 0.17$ & ns \\
\hline HDL-C (mmol/li) & $1.41 \pm 0.05$ & $1.38 \pm 0.06$ & $1.43 \pm 0.06$ & ns \\
\hline $\mathrm{FT}_{4}(\mathrm{pmol} / \mathrm{l})(9.01-24.45)$ & $14.5 \pm 0.67$ & $14.0 \pm 0.70$ & $14.9 \pm 0.42$ & $\mathrm{~ns}$ \\
\hline $\mathrm{FT}_{3}(\mathrm{pmol} / \mathrm{l})(4.00-7.70)$ & $5.06 \pm 0.26$ & $4.92 \pm 0.31$ & $5.21 \pm 0.19$ & ns \\
\hline TSH (mIU/I) (0.4-4.7) & $7.32 \pm 0.64$ & $7.25 \pm 0.69$ & $2.52 \pm 0.30^{\star}$ & $<0.001$ \\
\hline $\operatorname{Tg}-\mathrm{Ab}(\mathrm{U} / \mathrm{ml})(<0.4)$ & $68.9 \pm 91$ & $70.8 \pm 86$ & Negative & ns \\
\hline TPO-Ab (U/ml) $(<0.3)$ & $290 \pm 324$ & $284 \pm 330$ & Negative & $\mathrm{ns}$ \\
\hline CRP (ng/ml) & $1032 \pm 398$ & $1019 \pm 410$ & $684 \pm 172$ & ns \\
\hline $\mathrm{BaPWV}(\mathrm{cm} / \mathrm{s})$ & $1776.7 \pm 86.0$ & $1742.2 \pm 100.4$ & $1414.2 \pm 58.6^{*}$ & $<0.001$ \\
\hline PET/ET ratio & $3.08 \pm 0.11$ & $3.01 \pm 0.14$ & $3.02 \pm 0.09$ & ns \\
\hline
\end{tabular}

Data are expressed as means \pm S.E.M. Differences in clinical factors among L-T 4 -treated patients and placebo patients and normal controls were examined using the two-tailed multiple $t$-test with Bonferroni correction. ns, not significant among the three groups; ${ }^{*}$, significant compared with the other groups; Systolic BP, systolic blood pressure; Diastolic BP, diastolic blood pressure; Total chol, Total cholesterol; LDL-C, LDL cholesterol; HDL-C, HDL cholesterol; Tg-Ab, antithyroglobulin antibody; TPO-Ab, antithyroid peroxidase antibody; CRP, C-reactive protein; baPWV, brachial-ankle pulse wave velocity; PET/ET ratio, preejection time/ejection time ratio. 


\section{Effect of $L-T_{4}$ replacement therapy on baPWV and clinical variables in female subclinical hypothyroid patients}

$\mathrm{L}-\mathrm{T}_{4}$ replacement therapy restored the serum levels of TSH to within the normal range $(2.7 \pm 0.21 \mathrm{mIU} / \mathrm{l})$ and also produced a significant decrease in baPWV from $1776.7 \pm 86.0$ to $1674.3 \pm 79.2 \mathrm{~cm} / \mathrm{s}(P=0.006$; Table 2). No significant change in BMI, SBP, DBP, pulse pressure, pulse rate, PET/ET ratio, serum total cholesterol, LDL and HDL cholesterol, triglyceride, CRP or serum $\mathrm{FT}_{4}$ and $\mathrm{FT}_{3}$ was observed. Placebo patients and normal controls (data not shown) showed no significant changes in all of these clinical variables after 5 months. Individual changes in baPWV in patients during $\mathrm{L}-\mathrm{T}_{4}$ replacement therapy are shown in Fig. 1. In $73.7 \%$ of the patients, baPWV decreased during therapy, but after normalization of thyroid function the mean baPWV was still higher than that of normal controls followed without medication for 5 months, as shown in Table 3 $(P<0.001)$. Patients receiving placebo and normal controls showed no significant changes in baPWV after 5 months (Table 3).

\section{Correlation of changes in baPWV with those in TSH and $\mathrm{FT}_{4}$ in patients treated with $\mathrm{L}_{-} \mathrm{T}_{4}$}

The changes in baPWV were not correlated with those in TSH and $\mathrm{FT}_{4}$ in patients who received $\mathrm{L}-\mathrm{T}_{4}$ replacement therapy $(\rho=-0.09 ; P=0.69, \rho=0.15$; $P=0.54$ respectively), although the change in $\mathrm{FT}_{4}$ was not significant. Moreover, no significant correlation was found between the changes in baPWV and $\mathrm{FT}_{4}$ values after treatment $(\rho=-0.05 ; P=0.82)$.

\section{Correlation of the change in baPWV with baseline factors in patients treated with ${ }^{-} \mathrm{T}_{4}$}

The change in baPWV was significantly and negatively correlated with age and baseline pulse pressure in patients treated with $\mathrm{L}_{-} \mathrm{T}_{4}(\rho=-0.611 ; P=0.0034$, $\rho=-0.538 ; P=0.0096$ respectively), but not with the other baseline factors including levels of $\mathrm{TSH}, \mathrm{FT}_{4}$, antithyroglobulin or antithyroid peroxidase antibodies (data not shown).

\section{Factors associated with the change in baPWV in patients treated with $L-T_{4}$}

Stepwise multiple regression analysis of the association of baseline clinical variables with changes in baPWV in patients treated with $\mathrm{L}-\mathrm{T}_{4}$ was performed. The analysis included baseline age and pulse pressure, which showed a significant correlation with the change in baPWV. However, these parameters failed to be associated with the change in baPWV during normalization of thyroid function (data not shown).

\section{Discussion}

In the present study, we demonstrated that sustained normalization of thyroid function caused a significant decrease in baPWV in female subclinical hypothyroid patients with autoimmune chronic thyroiditis. This finding suggests that this therapy may be beneficial for the improvement of arterial stiffening in female subclinical hypothyroid patients with autoimmune thyroiditis.

Since baPWV is not only a parameter of arterial stiffness (9) but also an independent predictor of cardiovascular mortality (10), a decrease in baPWV

Table 2 Clinical variables in $\mathrm{L}_{-} \mathrm{T}_{4}$-treated patients after normalization of thyroid function and in patients receiving placebo after 5 months.

\begin{tabular}{|c|c|c|c|c|}
\hline & $\mathrm{L}-\mathrm{T}_{4}$-treated patients & $\boldsymbol{P}$ & Placebo patients & $\boldsymbol{P}$ \\
\hline Body mass index $\left(\mathrm{kg} / \mathrm{m}^{2}\right)$ & $21.8 \pm 0.48$ & ns & $22.1 \pm 0.50$ & ns \\
\hline Systolic BP (mmHg) & $128.8 \pm 3.8$ & ns & $132.2 \pm 3.5$ & ns \\
\hline Pulse pressure (mmHg) & $56.0 \pm 2.8$ & ns & $57.5 \pm 2.3$ & ns \\
\hline Pulse rate (/min) & $70.1 \pm 1.7$ & ns & $70.3 \pm 2.0$ & ns \\
\hline Total chol (mmol/l) & $5.19 \pm 0.16$ & ns & $5.33 \pm 0.24$ & ns \\
\hline Triglyceride $(\mathrm{mmol} / \mathrm{l})$ & $1.50 \pm 0.16$ & ns & $1.38 \pm 0.14$ & ns \\
\hline LDL-C (mmol/l) & $3.14 \pm 0.29$ & ns & $3.36 \pm 0.19$ & $\mathrm{~ns}$ \\
\hline $\mathrm{HDL}-\mathrm{C}(\mathrm{mmol} / \mathrm{l})$ & $1.41 \pm 0.08$ & ns & $1.39 \pm 0.06$ & ns \\
\hline TSH (mIU/l) & $2.7 \pm 0.21$ & $<0.0001$ & $7.01 \pm 0.67$ & ns \\
\hline $\mathrm{FT}_{4}(\mathrm{pmol} / \mathrm{l})$ & $16.0 \pm 0.69$ & ns & $14.8 \pm 0.72$ & ns \\
\hline $\mathrm{FT}_{3}(\mathrm{pmol} / \mathrm{l})$ & $5.14 \pm 0.26$ & ns & $5.12 \pm 0.27$ & $\mathrm{~ns}$ \\
\hline $\mathrm{CRP}(\mathrm{ng} / \mathrm{ml})$ & $1012 \pm 384$ & ns & $1024 \pm 402$ & ns \\
\hline $\mathrm{BaPWV}(\mathrm{cm} / \mathrm{s})$ & $1674.3 \pm 79.2$ & 0.006 & $1790.9 \pm 112.0$ & $\mathrm{~ns}$ \\
\hline PET/ET ratio & $3.11 \pm 0.12$ & ns & $3.08 \pm 0.14$ & ns \\
\hline
\end{tabular}

Data are expressed as means \pm S.E.M. The differences of means before and after normalization of thyroid function in L-T ${ }_{4}$-treated patients and between baseline and after 5 months in placebo patients were assessed by a two-tailed Student's $t$-test for paired data. ns, not significant; Systolic BP, systolic blood pressure; Diastolic BP, diastolic blood pressure; Total chol, Total cholesterol; LDL-C, LDL cholesterol; HDL-C, HDL cholesterol; baPWV, brachial-ankle pulse wave velocity; PET/ET ratio, pre-ejection time/ejection time ratio; CRP, C-reactive protein. 


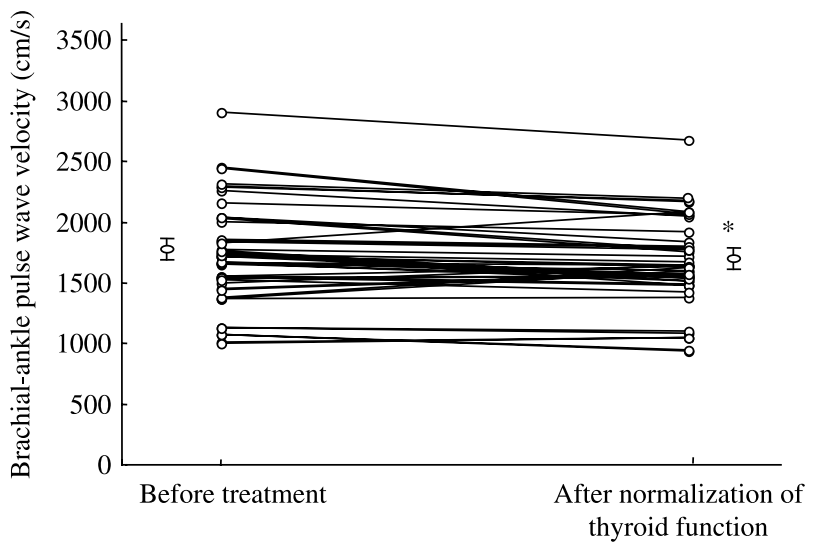

Figure 1 Individual changes in brachial-ankle pulse wave velocity (baPWV) before and after restoration of euthyroidism by $\mathrm{L}^{\mathrm{T}} \mathrm{T}_{4}$ replacement therapy in female subclinical hypothyroid patients. BaPWV decreased significantly from $1776.7 \pm 86.0$ to $1674.3 \pm$ $79.2 \mathrm{~cm} / \mathrm{s}(P=0.006)$.

means improvement in arterial stiffness and may diminish the risk of cardiovascular disease. However, the limitation of PWV measurement is that no comparison of baPWV with structural arterial change had thus far been reported.

It remains unclear whether subclinical hypothyroidism is a risk factor for atherosclerosis and treatment with $\mathrm{L}^{-} \mathrm{T}_{4}$ reverses this risk (24-26). Monzani et al. reported that subclinical hypothyroid patients have greater IMT, a clinically useful indicator of arterial thickening, and that $\mathrm{L}_{-} \mathrm{T}_{4}$ treatment improves IMT in these patients (7). There is no clear evidence that subclinical hypothyroidism causes clinical heart disease, but subclinical hypothyroidism is associated with impairment of several cardiac parameters (27) and manifests itself as a left ventricular dysfunction at rest and systolic and diastolic dysfunction during effort, which might explain why subclinical hypothyroidism leads to an enhanced risk for atherosclerosis and myocardial infarction (8). These cardiovascular

Table 3 Changes in brachial-ankle pulse wave velocity before and after normalization of thyroid function in patients treated with $\mathrm{L}-\mathrm{T}_{4}$, and between baseline and after 5 months in patients receiving placebo and in normal controls.

\begin{tabular}{lccc}
\hline & $\begin{array}{c}\text { BaPWV before } \\
\text { treatment or } \\
\text { at baseline } \\
(\mathrm{cm} / \mathrm{s})\end{array}$ & $\begin{array}{c}\text { BaPWV after } \\
\text { normalization } \\
\text { of thyroid } \\
\text { function or after } \\
\mathbf{5} \text { months }(\mathrm{cm} / \mathrm{s})\end{array}$ & \multicolumn{1}{c}{$\boldsymbol{P}$} \\
\hline $\mathrm{L}-\mathrm{T}_{4}$ treatment & $1776.7 \pm 86.0$ & $1674.3 \pm 79.2$ & 0.006 \\
Placebo & $1742.2 \pm 100.4$ & $1790.9 \pm 112.0$ & $\mathrm{~ns}$ \\
Normal controls & $1414.2 \pm 58.6$ & $1432.6 \pm 60.4$ & $\mathrm{~ns}$ \\
\hline
\end{tabular}

Data are expressed as means \pm S.E.M. The difference between the mean values before treatment and after restoration of normal thyroid function or between baseline and after 5 months was assessed by a two-tailed Student's $t$-test for paired data. ns, not significant; baPWV, brachial-ankle pulse wave velocity. abnormalities have been shown to regress with $\mathrm{L}_{-} \mathrm{T}_{4}$ therapy (28). In our previous investigation of the change in baPWV in subclinical hypothyroid patients without thyroiditis who were treated with $\mathrm{L}_{-} \mathrm{T}_{4}$, the proportion of patients showing a reduced baPWV was higher in females $(67.7 \%)$ than in males (36.4\%) (12), although not in a placebo-controlled study. The present findings also suggest that subclinical hypothyroidism, especially with high baseline baPWV, should be treated to restore normal thyroid function for prevention of atherosclerosis, although we note that the study was restricted to female patients with autoimmune chronic thyroiditis.

The lack of correlation of the change in baPWV with either baseline levels of serum TSH and $\mathrm{FT}_{4}$ or changes during $\mathrm{L}_{-} \mathrm{T}_{4}$ replacement therapy suggests that thyroid hormone might affect baPWV through indirect mechanisms, rather than directly. Moreover, no significant correlation of baPWV was found with autoimmune antibodies or CRP for either baseline levels or changes during $\mathrm{L}_{-} \mathrm{T}_{4}$ replacement therapy (data not shown), suggesting that inflammatory responses caused by autoimmune thyroiditis did not contribute to an increase in baPWV observed in subclinical hypothyroid patients with Hashimoto's disease. In our and other reports in overt hypothyroidism, CRP has been associated with arterial stiffness $(29,30)$. The elevation of CRP in subclinical hypothyroidism is controversial (31) and in this study serum level of CRP exhibited no significant difference between subclinical hypothyroid patients and normal controls. The stepwise multiple regression model failed to find independent factors associated with the change in baPWV, indicating the presence of other factors in female subclinical hypothyroidism. Therefore, further data are required to determine the significance of other factors associated with arterial stiffness in overt hypothyroidism besides CRP, and to elucidate the mechanism through which subclinical hypothyroidism may increase arterial stiffening.

The exact reason for persistently elevated baPWV after normalization of thyroid function is unclear. Since advanced atherosclerosis may not be completely reversible, arterial stiffness could not be improved to a normal level. In addition, short study period may be another reason.

The study period of about 5 months is slightly shorter than the previous 6-month studies of changes in cardiac function or central arterial stiffness by $\mathrm{L}_{-} \mathrm{T}_{4}$ replacement therapy in overt or subclinical hypothyroidism $(8,32)$. However, evaluation of the effect of various antihypertensive drugs on baPWV showed that only 3 months were required for a significant reduction in baPWV (the age of the cohort was 71.0 \pm 3.0$)(13)$, suggesting the high sensitivity and clinical significance of baPWV measurements over a short time period; even statins reduce baPWV markedly for only 6 months in diabetes (the age of the cohort was 61.7 \pm 7.2 ) (33). However, 
baPWV after restoration of normal thyroid function in patients treated with $\mathrm{L}-\mathrm{T}_{4}$ was still higher than that of normal subjects followed for 5 months. The length of euthyroid state was quite short $(7.46 \pm 0.69$ weeks), and therefore this may be the reason for lack of normalization of baPWV. Hence, it is of importance to determine whether a longer period of $\mathrm{L}-\mathrm{T}_{4}$ replacement therapy for patients with subclinical hypothyroidism might restore baPWV to the normal range. Although the population is little until now, after more than 3 years of euthyroid state some of the enrolled patients attained age-matched normal standard value of baPWV (during followup study).

In our protocol for $\mathrm{L}-\mathrm{T}_{4}$ replacement therapy for subclinical hypothyroidism, the initial daily dose of $\mathrm{L}-\mathrm{T}_{4}$ of $12.5 \mu \mathrm{g}$ was increased serially to $18.75 \mu \mathrm{g} /$ day, $25 \mu \mathrm{g} /$ day, and maximally to $37.5 \mu \mathrm{g} /$ day until TSH decreased within normal range. Serum $\mathrm{FT}_{4}, \mathrm{FT}_{3}$, and TSH were checked every 4 weeks during therapy. None of the patients required withdrawal of $\mathrm{L}^{-\mathrm{T}_{4}}$ due to side effects such as arrhythmia, angina pectoris, and hypertension. Moreover, they did not develop suppression of serum TSH values below its lower limit. Therefore, this therapeutic regimen appears to be effective in avoidance of cardiovascular side effects of $\mathrm{L}-\mathrm{T}_{4}$ in the treatment of subclinical hypothyroidism.

In summary, our results demonstrate that the sustained normalization of thyroid function during $\mathrm{L}-\mathrm{T}_{4}$ replacement therapy significantly decreases baPWV in female subclinical hypothyroid patients with autoimmune chronic thyroiditis. This finding suggests that $\mathrm{L}-\mathrm{T}_{4}$ replacement therapy has beneficial effects in these patients, suggesting the improvement of arterial stiffening.

\section{Declaration of interest}

We declare that there is no conflict of interest that could be perceived as prejudicing the impartiality of the scientific work reported.

\section{Funding}

This research did not receive any specific grant from any funding agency in the public, commercial or not-for-profit sector.

\section{References}

1 Evered DC, Tunbridge WM, Hall R, Appleton D, Brewis M, Clark F, Manuel P \& Young E. Thyroid hormone concentrations in a large scale community survey. Effect of age, sex, illness and medication. Clinica Chimica Acta $197883223-229$.

2 Cappola AR, Fried LP, Arnold AM, Danese MD, Kuller LH, Burke GL, Tracy RP \& Ladenson PW. Thyroid status, cardiovascular risk, and mortality in older adults. Journal of the American Medical Association 2006295 1033-1041.

3 Singh S, Molnar J \& Arora R. Association between subclinical hypothyroidism and coronary heart disease in 2006. International Journal of Cardiology 2008125 41-48.
4 Luboshitzky R, Aviv A, Herer P \& Lavie L. Risk factors for cardiovascular disease in women with subclinical hypothyroidism. Thyroid $200212421-425$.

5 Althaus BU, Staub JJ, Ryff-De Leche A, Oberhansli A \& Stahelin HB. LDL/HDL-changes in subclinical hypothyroidism: possible risk factors for coronary heart disease. Clinical Endocrinology $1998 \mathbf{2 8}$ 157-163.

6 Pearce EN. Hypothyroidism and dyslipidemia: modern concepts and approaches. Current Cardiology Reports 20046 451-456.

7 Monzani F, Caraccio N, Kozakowa M, Dardano A, Vittone F, Virdis A, Taddei S, Palombo C \& Ferrannini E. Effect of levothyroxine replacement on lipid profile and intima-media thickness in subclinical hypothyroidism: a double-blind, placebocontrolled study. Journal of Clinical Endocrinology and Metabolism 200489 2099-2106.

8 Monzani F, Di Bello V, Caraccio N, Bertini A, Giorgi D, Giusti C \& Ferrannini EJ. Effect of levothyroxine on cardiac function and structure in subclinical hypothyroidism: a double blind, placebocontrolled study. Journal of Clinical Endocrinology and Metabolism 200186 1110-1115.

9 Yamashina A, Tomiyama H, Takeda K, Tsuda H, Arai T, Hirose K, Koji Y, Hori S \& Yamamoto Y. Validity, reproducibility, and clinical significance of noninvasive brachial-ankle pulse wave velocity measurement. Hypertension Research 200225 359-364.

10 Measume S, Benetos A, Henry OF, Rundnichi A \& Safar ME. Aortic pulse wave velocity predicts cardiovascular mortality in subjects $>70$ years of age. Arteriosclerosis, Thrombosis, and Vascular Biology $2001212046-2050$.

11 Nagasaki T, Inaba M, Kumeda Y, Hiura Y, Shirakawa K, Yamada S, Henmi Y, Ishimura E \& Nishizawa Y. Increased pulse wave velocity in subclinical hypothyroidism. Journal of Clinical Endocrinology and Metabolism 200591 154-158.

12 Nagasaki $T$, Inaba $M$, Yamada $\mathrm{S}$, Kumeda $\mathrm{Y}$, Hiura $\mathrm{Y}$ \& Nishizawa Y. Changes in brachial-ankle pulse wave velocity in subclinical hypothyroidism during normalization of thyroid function. Biomedicine and Pharmacotherapy 200761 482-487.

13 Takami T \& Shigemasa M. Efficacy of various antihypertensive agents as evaluated by indices of vascular stiffness in elderly hypertensive patients. Hypertension Research 200326 609-614.

14 Miura S, Tanaka E, Mori A, Toya M, Takahashi K, Nakahara K, Ohmichi M \& Kurachi H. Hormone replacement therapy improves arterial stiffness in normotensive postmenopausal women. Maturitas 200345 293-298.

15 Koshiyama H, Tanaka S \& Minamikawa J. Effect of calcium channel blocker amlodipine on the intima-media thickness of carotid arterial wall in type 2 diabetes. Journal of Cardiovascular Pharmacology 199933 894-896.

16 Mercuri M, Bond MG, Sirtori CR, Veglia F, Crepaldi G, Feruglio FS, Descovich G, Ricci G, Rubba P, Mancini M, Gallus G, Bianchi G, D'Alò G \& Ventura A. Pravastatin reduces carotid intima-media thickness progression in an asymptomatic hypercholesterolemic Mediterranean population:the carotid atherosclerosis Italian ultrasound study. American Journal of Medicine $1996 \mathbf{1 0 1}$ 627-634.

17 Migdalis IN, Varvarigos N, Charalabides J, Leontiades E, Gerolimou B, Mantzara F \& Karmaniolas K. Effects of buflomedil on early carotid atherosclerosis in Type 2 diabetic patients. International Angiology 200120 126-130.

18 Koshiyama H, Nakamura Y, Tanaka S \& Minamikawa J. Decrease in carotid intima-media thickness after 1-year therapy with etidronate for osteopenia associated with type 2 diabetes. Journal of Clinical Endocrinology and Metabolism 200085 2793-2796.

19 Friedewald WT, Levy RI \& Fredrickson DS. Estimation of the concentration of low-density lipoprotein in plasma without use of preparative ultracentrifuge. Clinical Chemistry 197218499.

20 Liuzzo G, Biasucci LM, Gallimore JR, Grillo RL, Rebuzzi AG, Pepys MB \& Maseri A. The prognostic value of C-reactive protein and serum amyloid a protein in severe unstable angina. New England Journal of Medicine 1994331 417-424. 
21 Girgensohn S, Liedtke R, Balzer G, Castor S \& Hauser M. Performance of the ACS: Centaur ${ }^{\mathrm{TM}}$ high-capacity, random-access immunoassay system. Clinical Laboratory 199743 975-983.

22 Arai T, Kurashima C, Utsuyama M, Sawabe M \& Ito H. Measurement of anti-thyroglobulin and anti-thyroid peroxidase antibodies using highly sensitive radioimmunoassay: an effective method for detecting asymptomatic focal lymphocytic thyroiditis in the elderly. Endocrine Journal 200047 575-582.

23 Kelly R, Hayward C, Avolio A \& O'Rourke M. Noninvasive determination of age-related changes in the human arterial pulse. Circulation $1989 \mathbf{8 0} 1652-1659$.

24 McDermott MT \& Ridgway EC. Subclinical hypothyroidism is mild thyroid failure and should be treated. Journal of Clinical Endocrinology and Metabolism 200186 4585-4590.

25 Meier C, Staub JJ, Roth CB, Gueglielmetti M, Kunz M, Miserez AR, Drewe J, Huber P, Herzog R \& Muller B. TSH controlled L-thyroxine therapy reduces cholesterol levels. Journal of Clinical Endocrinology and Metabolism $2001 \mathbf{8 6} 4860-4866$.

26 Chu JW \& Crapo LM. The treatment of subclinical hypothyroidism is seldom necessary. Journal of Clinical Endocrinology and Metabolism $2001864591-4599$.

27 Kahaly GJ. Cardiovascular and atherogenic aspects of subclinical hypothyroidism. Thyroid 200010 665-679.

28 Biondi B \& Klein I. Hypothyroidism as a risk factor for cardiovascular disease. Endocrine 200424 1-13.
29 Nagasaki T, Inaba M, Shirakawa K, Hiura Y, Tahara H, Kumeda Y, Ishikawa T, Ishimura E \& Nishizawa Y. Increased levels of C-reactive protein in hypothyroid patients and its correlation with arterial stiffness in the common carotid artery. Biomedicine and Pharmacotherapy 200761 167-172.

30 Christ-Crain M, Meier C, Guglielmetti M, Huber PR, Riesen W, Staub JJ \& Müller B. Elevated C-reactive protein and homocysteine values: cardiovascular risk factors in hypothyroidism? A crosssectional and a double-blind, placebo-controlled trial Atherosclerosis $2003 \mathbf{1 6 6} 379-386$.

31 Lee WY, Suh JY, Rhee EJ, Park JS, Sung KC \& Kim SW. Plasma CRP, apolipoprotein A-1, apolipoprotein B and Lpa levels according to thyroid function status. Archives of Medical Research 200435 540-545.

32 Obuobie K, Smith J, Evans LM, John R, Davies JS \& Lazarus JH. Increased central arterial stiffness in hypothyroidism. Journal of Clinical Endocrinology and Metabolism $2002874662-4666$.

33 Shinohara K, Shoji T, Kimoto E, Yokoyama H, Fujiwara S, Hatsuda S, Maeno T, Shoji T, Fukumoto S, Emoto M, Koyama H \& Nishizawa Y. Effect of atorvastatin on regional arterial stiffness in patients with type 2 diabetes mellitus. Journal of Atherosclerosis and Thrombosis 200512 205-210.

Received 28 October 2008

Accepted 15 December 2008 